\title{
Characterization of DuPont photopolymer: determination of kinetic parameters in a diffusion
}

\section{model}

Vincent Moreau, Yvon Renotte, and Yves Lion

- Applied Optics

- Vol. 41,

- Issue 17,

- pp. 3427-3435

- (2002)

- $\quad$ https://doi.org/10.1364/AO.41.003427

\section{Abstract}

We investigate the recording dynamics of Omnidex photopolymer film from DuPont. We use a reviewed version of the diffusion model proposed by Zhao and Mouroulis [J. Mod. Opt. 41, 1929 (1994)] in order to describe the recording response that combined photopolymerization and free-monomer diffusion process. Two different experiments are detailed that lead to the determination of material kinetic parameters. These values are introduced in the numerical model to provide quantitative simulations of a grating formation under various holographic exposures. Theoretical results are experimentally checked as a validation of the model. We extend its applications to several secondary investigations, such as volume-shrinkage influence on refractive-index distribution and spectral selectivity of reflection gratings. This study improves the understanding of the recording process and consequently allows to build more accurate holographic components in this material to be built.

(c) 2002 Optical Society of America 